\title{
REVIEW
}

\section{Male infertility microsurgical training}

\begin{abstract}
Akanksha Mehta and Philip S Li
Microsurgical training is imperative for urologists and clinical andrologists specializing in male infertility. Success in male infertility microsurgery is heavily dependent on the surgeon's microsurgical skills. Laboratory-based practice to enhance microsurgical skills improves the surgeon's confidence, and reduces stress and operating time, benefiting both the patient and the surgeon. This review provides guidelines for setting up a microsurgical laboratory to develop and enhance microsurgical skills using synthetic and animal models. The role of emerging techniques, such as robotic-assisted microsurgery, is also discussed.
\end{abstract}

Asian Journal of Andrology (2013) 15, 61-66; doi:10.1038/aja.2012.86; published online 19 November 2012

Keywords: laboratory; male infertility; microsurgery; training; vasoepididymostomy; vasovasostomy

\section{INTRODUCTION}

Microsurgical training is imperative for urologists and clinical andrologists specializing in male infertility. However, developing good microsurgical skills can be challenging for any surgeon, because of distinct differences between microsurgical and conventional open surgical techniques. The use of an operating microscope dramatically changes the scale of surgery, and alters the surgeon's spatial perception. The use of fine microsurgical instruments and suture materials introduces novel ergonomic considerations. Coordination, manual dexterity and steadiness of movement under the microscope require practice and time to achieve. Success in male infertility microsurgery is heavily dependent on surgical skill. Patient outcomes are often not apparent for months after the procedure, until sperm appear in the ejaculate. Male infertility microsurgery may be the only surgical procedure of which surgeon may not know the outcome at the end of the case, until confirmation from a laboratory report, such as a semen analysis, is obtained. Male infertility microsurgery, therefore, ranks the most technically and mentally challenging surgical procedures.

Surgical education has traditionally been built on the Halstedian apprenticeship model, where experts teach skills to surgeons in training using real patients. ${ }^{1}$ But increasing pressures on operating room time and resources have called the role of the operating room into question as the exclusive venue for teaching surgical skills. ${ }^{2}$ There is increasing interest in the development of surgical skills training laboratories, on the part of various surgical specialties. Recently, a prospective study of 50 surgical residents randomized to hands-on laboratory versus didactic training alone, demonstrating that laboratory-based technical skills training resulted in significantly better surgical performance. ${ }^{3}$ Laboratory training was also associated with better retention of technical skills by novice surgeons over a 4 -month follow-up period. ${ }^{3}$

Cognitive and technical competency in microsurgery are essential before entering the operating room, and are best established through repeated practice in a microsurgical training laboratory. The aim of this article is to provide guidelines for setting up a microsurgical laboratory and to review basic microsurgical skills using synthetic and animal models. The role of emerging techniques, such as robotic-assisted microsurgery, is also discussed.

\section{THE MICROSURGERY LABORATORY}

Establishing a microsurgical laboratory need not be expensive, and does not require a lot of elaborate equipment beyond an adequate operating microscope and select microsurgical instruments. Synthetic practice materials, such as suturing practice cards, and silicone tubing are inexpensive models for developing basic microsurgical skills. ${ }^{4}$ Advanced skills for vasovasostomy and vasoepididymostomy can be acquired using human vas segments, or small animals such as rats. While live animal surgery provides a high-fidelity model for microsurgical training and research, it can be expensive and requires access to an established animal care facility.

\section{Operating microscope}

There are a variety of microscopes available for use in the laboratory. The characteristics of a good operating microscope include bright illumination, a smooth focusing system and ease of operation. For male infertility microsurgery, a magnification range between $\times 10$ and $\times 25$ is sufficient. Either a motorized or a manual zoom and focus system may be used for training purposes. The basic components of an operating microscope consist of the objective lens, eyepieces, binocular tube and microscope body, which houses the magnification changer. The objective lens determines the focal length or working distance. A 200-mm objective length is usually used for male infertility microsurgery, which focuses on the surgical field $200 \mathrm{~mm}$ away from the objective lens. Correct set-up of the objective length minimizes physical strain to the surgeon, and allows for easy handing of instruments under the microscope.

The operating microscope should be secured to a solid and stable working table, with workspace dimensions of at least 30 inches $\times 24$ inches. Ensuring a table height of at least 30 inches allows for comfortable placement of the operator's knees under the table. 
In our microsurgical laboratory, we use the Zeiss OPMI/S3 and Zeiss OPMI Cs/S4 surgical microscope systems (Carl Zeiss AG, Oberkochen, Germany) with binocular microscopes. ${ }^{5}$ Two binocular heads allow for an instructor to observe and provide guidance and feedback to the trainee.

\section{Microsurgical instruments}

Although a variety of specialized microsurgical instruments are commercially available, the majority of urological microsurgical procedures can be performed using the select microsurgical instruments listed below.

\section{Microsurgical set.}

1. Non-locking needle-holder with a rounded, fine-curved tip ( 13.5 or $15 \mathrm{~cm}$ in length);

2. Straight, fine-tip forceps with suture platform $(13.5$ or $15 \mathrm{~cm}$ in length);

3. Straight, fine tissue forceps with teeth ( 13.5 or $15 \mathrm{~cm}$ in length);

4. Curved, blunt-tip dissecting scissors;

5. Sharp, Iris scissors;

6. Angled vessel dilator with a slender, tapered tip;

7. Vas approximator clamp for vasovasostomy and vasoepididymostomy, such as the Goldstein microspike approximator, available in different sizes to accommodate various luminal diameters, as well as end-to-end and end-to-side configurations (ASSI Inc., Westbury, NY, USA);

8. Microsurgical bipolar cautery with fine-tipped forceps;

9. Non-sterile Sharpoint microsurgical single-armed 9-0 and singleor double-armed 10-0 nylon sutures (Angiotech Pharmaceutical Inc., Vancouver, BC, Canada);

10. Sharpoint microsurgical suturing practice cards (Angiotech Pharmaceutical Inc.);

11. Microtip surgical marking pen, such as Kendall Devon fine-tip surgical skin marker (KDL-311145942) (Devon Industries, Buffalo, NY, USA).

The following additional surgical supplies are useful for establishing a fully equipped microsurgical laboratory, to allow for animal surgery as well.

Non-microsurgical set.

1. Basic set of instruments for small animal surgery, including small needle holder, smooth and toothed forceps, suture scissors, curved dissecting scissors, and clip applier;

2. Operating board, at least $35 \mathrm{~cm} \times 35 \mathrm{~cm}$ in size;

3. Soft silicone tubing or preserved segments of vas deferens;

4. Tape for fixing practice objects to operating board;

5. Ten milliliter of syringe and attached 27-guage angiocatheter for irrigation, with normal saline or lactated Ringer's solution;

6. Non-reflective drape to cover operative field and provide a satisfactory background, such as blue-colored paper drapes or towels;

7. Spasmolytics, such as $1 \%$ or $2 \%$ lidocaine hydrochloride $\left(20 \mathrm{mg} \mathrm{ml}^{-1}\right)$;

8. Heparin sulfate solution (100-150 units $\mathrm{ml}^{-1}$ ), for use during vasovasal re-anastomosis in animal surgery;

9. Hemolytic enzyme cleaning solution, such as Haemo-Sol (Haemo-Sol Inc., Baltimore, MD, USA);

10. Storage trays for microsurgical instruments;

11. Surgical instrument demagnetizer.

Good performance in microsurgery requires well-maintained microsurgical instruments. Working with damaged instruments can be laboriously time consuming and frustrating, for both novices and experienced microsurgeons. Microsurgical instruments should be handled with care, and stored in a specially designed instrument case. Each instrument should be examined under the microscope prior to use. If necessary, instrument tips can be sharpened and repaired using Arkansas oil stone or emery paper.

Following use, instruments should be soaked in a hemolytic enzyme solution and then rinsed with water for careful cleaning. Damage to instruments is most likely to occur during the cleaning process. Once dry, the fine instrument tips should be protected by plastic covers or segments of silicone tubing prior to storage. Contacting with other metal objects can cause the instruments to become magnetized. An inexpensive surgical instrument demagnetizer can be used to address this problem.

\section{Basic preparation}

In order to perform well, it is essential that the microsurgeon be familiar with his or her operating microscope and create a comfortable working environment. Developing accurate microsurgical techniques also requires extensive patience and frequent practice until basic skills are mastered. Simple strategies such as adequate rest prior to a practice session, avoidance of heavy lifting and minimization of mental stress can maximize the benefit from each practice session.

Once in the laboratory, an ergonomic sitting position at the working table, with the use of towels or foam to support the arms and hands, as necessary, is necessary to maintain surgeon comfort. Maximum illumination with sharp focus should be utilized under the microscope. Correcting the focus at the highest magnification ensures focus at all lower magnifications. The interocular distance of the eyepieces should be adjusted until a single image is visible through the binocular scope. The appropriate focal distance should be used while operating under the microscope.

The level of magnification should be adjusted as necessary during practice, as it is during surgical procedures. Low magnification should be used for tissue dissection and manipulation of the needle and suture materials. In contrast, high magnification is needed for preparation of the vasal ends or the epididymal tubules for re-anastomosis, and for passage of the needle through the lumen of the vas or epididymis. Following suture placement, surgical knots are best tied under low magnification.

\section{SUTURING TECHNIQUE}

Any introduction to microsurgical techniques must begin with a discussion of proper hand positioning and instrument handling, before skills such as suture placement and knot-tying can be discussed. Hand tremor can be a significant problem when working under the microscope, even for experienced microsurgeons. Proper hand support, with folded surgical towels to support the hand and forearm, is important to minimize tremor and hand strain. Most microsurgical tasks, including suturing and knot tying, require only a slight movement of the fingers. Keeping the remainder of the hand still, with the thumb, index and middle finger supporting each other, further serves to limit tremors.

While many surgeons develop their own preferred way of holding microsurgical instruments, the pencil-holding position, with the instrument resting between the thumb and index finger, is recommended (Figure 1). This position allows for controlled movement of the instrument while providing maximum ergonomic support for the surgeon's hand.

Urologic microsurgery utilizes fine 9-0 and 10-0 needles and suture material, which are easily damaged if held too firmly. Developing a secure but gentle touch is important to accurately control microsurgical instruments with minimal trauma to the suture materials. 


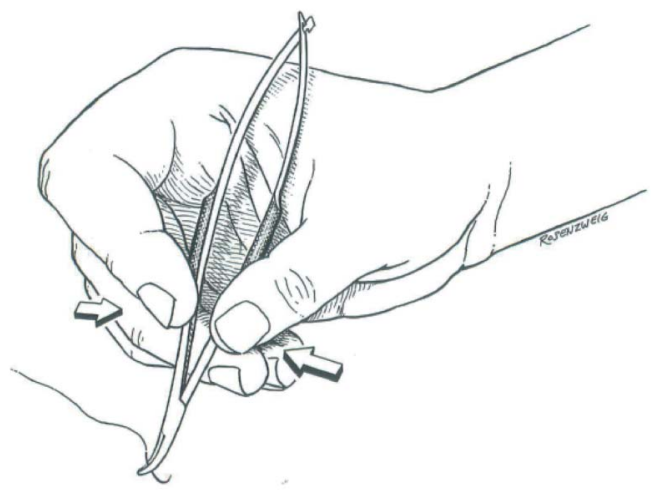

Figure 1 Pencil-holding position for manipulating microsurgical instruments.

Indeed, one mark of a good surgeon is the ability to preserve the original shape and condition of the suture needle throughout the operation. For maximal stability, the needle should be grasped by the needle holder at a point that is approximately one half to twothirds of the way away from the tip of the needle. Fine adjustments to the angle of the needle can be made by touching the needle with the tips of the forceps. The needle should always be manipulated under the optical magnification of the operating microscope.

The direction of the needle naturally depends on whether a forehand or backhand stroke is planned. For most individuals, mastering the forehand stroke is considerably easier. However, being able to effectively handle and rotate the needle in any direction is essential for a microsurgeon, and developing this basic skill alone entails considerable patience and practice.

\section{Microsuturing practice card}

After mastering manipulation of the needle with the needle holder and forceps, the next step is to learn basic microsurgical suturing technique. In our microsurgical laboratory, we use non-sterile singlearmed or double-armed 10-0 monofilament non-absorbable nylon sutures for practice, as well as for vaso-vasal and vaso-epididymal anastomoses. A practice suturing card, made of latex (Angiotech Pharmaceutical Inc.), is a simple and cost-effective tool for developing suturing skills under the microscope.

An incision is made on the card with a scalpel, with the goal of placing a series of interrupted stiches in order to close the incision. It is important to estimate the entrance and exit points prior to passing the needle through the card. These sites should be equidistant from the edge of the incision. The needle should pierce the latex 'tissue' perpendicularly, at a point that is approximately two-thirds the thickness of the tissue. The tips of the forceps should be used to apply gentle counter-pressure to help pass the needle through the tissue, one edge at a time. Movement of the surgeon's fingers must always follow the curve of the needle, in order to minimize trauma to both the tissue and the needle (Figure 2).

\section{Microsurgical knots}

Male infertility microsurgical procedures often involve challenging anastomoses between discrepant luminal diameters, thick muscular walls of the vas deferens on one hand, and delicate, thin walls of the epididymal tubules on the other. As a result, suture placement and knot tying can easily consume $40 \%-65 \%$ of the total operative time. ${ }^{5}$ The success of these procedures is greatly facilitated by the surgeon's ability to efficiently and securely tie microsurgical knots under the operating microscope.
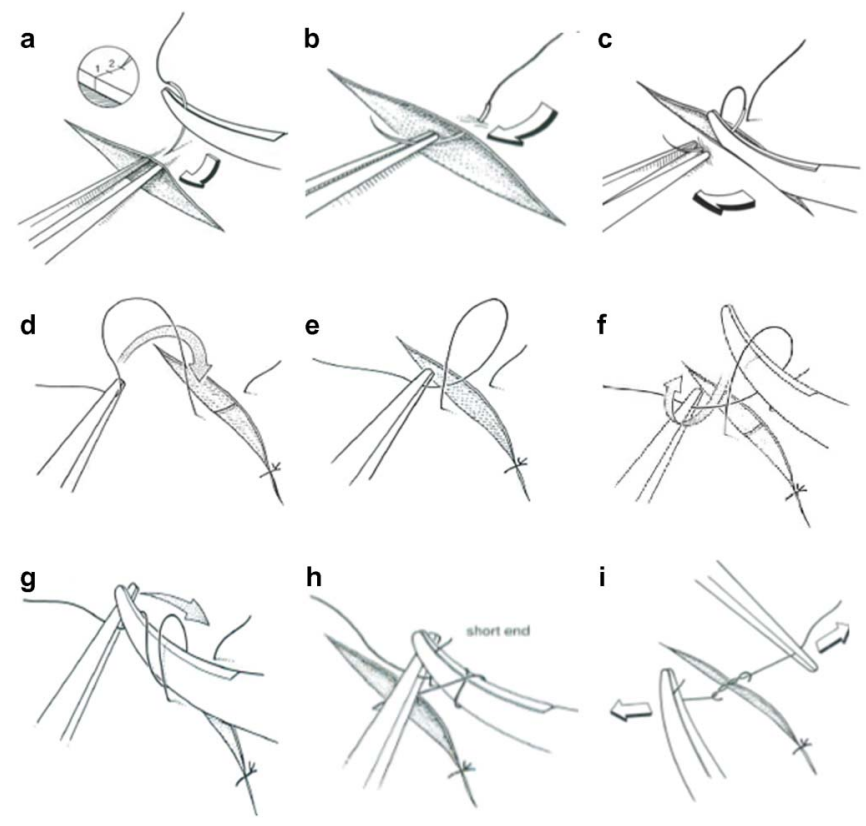

Figure 2 Step-wise completion of a microsurgical knot.

Given the complexity of the anastomoses in male infertility microsurgery, and the level of precision required, most microsurgeons prefer a double-throw surgeon's knot as the first knot, followed by two or more single-throw square knots, to prevent suture unraveling (Figure 2). The first knot should be tightened until the tissue edges are just approximated, but not strangulated. Repeated practice with knot-tying in the microsurgical laboratory is important to learn the optimum technique for looping the suture around the surgical instruments, the necessary length needed for the short end of the suture and appropriate amount of tension required to secure the knot without breaking the suture. Once the knot has been completed, the suture should be placed on gentle tension and cut under the microscope using sharp microscissors, leaving a length of $1-2 \mathrm{~mm}$, in order to prevent unraveling of the knots.

Online microsurgical videos are a useful educational resource for understanding and learning both basic suturing techniques, as well as more complex procedures in male infertility microsurgery. For more detail, the reader is referred to: www.maleinfertility.org.

\section{MODELS FOR MICROSURGICAL TRAINING: SILICONE TUBING, VASECTOMIZED SEGMENTS AND LIVE ANIMAL SURGERY}

Microsurgical vasovasostomy and vasoepididymostomy are amongst the most difficult anastomoses in microsurgery. Several different models for vasovasostomy and vasoepididymostomy training can be used in the laboratory, ranging from silicone tubing, to segments of human vas deferens and live animals. A good model should allow not only for practicing suture placement techniques, but also for practicing and optimizing the set-up of the surgical field to carry out these delicate anastomoses.

While most microsurgeons prefer the use of double-armed sutures for vasovasostomy in the operating room, these can be expensive for use in the microsurgical laboratory. Less expensive and more widely available single-armed sutures can be used for training purposes. Monoski et al. ${ }^{6}$ have also described an innovative technique for vasovasostomy and vasoepididymostomy using single-armed sutures. Mastery of vasal anastomoses using single-armed sutures may certainly be important for clinical settings where access to doublearmed suture is either unavailable or prohibitively expensive. 


\section{Silicone tubing}

Soft silicone tubing is an inexpensive and effective alternative to the use of human specimens and live animals. A 5- to $10-\mathrm{cm}$ length of medical grade silicone tubing (inner diameter 0.062 inch; outer diameter 0.0125 inch) can be used to practice both the one-layer and twolayer techniques for an end-to-end vasovasostomy. The one-layer technique, being simpler, has the advantage of allowing the trainee to focus on proper position, order, and suture placement for the anastomosis. The tubing is held and stabilized on the surgical field with a Microspike approximator, which, in turn, is fixed with tape to the surgical field to prevent its movement under the microscope. ${ }^{7}$ The tubing is divided with a scalpel between the arms of the approximator, to reveal its lumen, as well as its anterior and posterior walls.

Prior to commencing the anastomosis, it is important for the surgeon to estimate the number of interrupted stiches required to complete the anastomosis. Marking the location of these stiches using a microtip surgical marking pen allows the surgeon to separate the act of planning stitch placement from their execution, leading to greater concentration ability and accuracy. ${ }^{8}$ We strongly recommend that microsurgeons-in-training employ this additional step (Figure 3).

For the one-layer technique, the needle is placed perpendicularly at a point approximately twice the wall thickness from the cut edge of the tubing, in the forehand direction, from the outside of the tubing towards the lumen. Counter pressure applied by the forceps is used to guide the needle through the wall of the tubing, exiting in the lumen, between the tips of the forceps. The needle is carefully pulled through the wall of the tubing following its curvature, using successive, gentle motions. The needle is then perpendicularly passed through the opposite wall of the tubing, from the lumen to the outside, exiting at a point that is equidistant from the cut edge of the tubing. The suture is securely tied, as described above, in order to approximate the edges of the tubing. Another two to four evenly-spaced stitches are placed on the remaining anterior wall of the tubing. The approximator is then turned over, and folded in the opposite direction, to expose the posterior wall of the tubing. The identical procedure is repeated on the second side. Normally, a single layer of 8-12 interrupted nylon stiches are required to complete the anastomosis of the silicone tubing.

Preparation of the silicone tubing for the two-layer anastomosis is the same as that described for the one-layer technique. The inner layer of the tubing simulates the vasal mucosal layer. Two or three 10-0 nylon sutures are placed through the inner one half of the tubing wall to achieve mucosal approximation. If single-armed suture is used, the stiches are both placed in the forehand direction. In contrast, if double-armed suture is used, forehand and backhand stiches are required in order for the suture knots to be tied outside the lumen of the tubing.

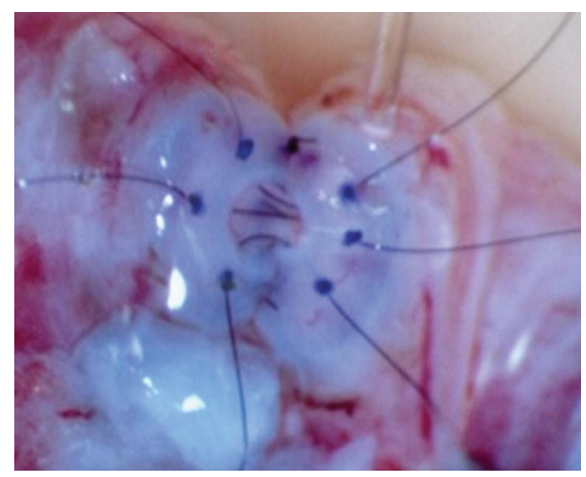

Figure 3 Vasovasostomy using microdot technique.
Once the mucosal stiches have been tied, single-armed 9-0 nylon suture is used to place interrupted stiches through the wall of the silicone tubing, without penetrating the lumen of the tubing, exactly in-between the previously placed mucosal stiches. This step simulates approximation of the vasal muscularis and adventitial layers. Once the anterior half of the anastomosis is complete, the approximator is turned over to expose the posterior wall of the tubing. The identical procedure is repeated on the second side. The two-layer anastomosis usually requires 6-8 interrupted 10-0 nylon stiches in the inner layer, and 8-12 interrupted 9-0 nylon stiches placed in the outer layer of the tubing.

\section{Vasectomy segments}

The use of vasectomy segments for microsurgical training was originally reported by Belker et al. ${ }^{9}$ in 1978. Long segments of vas can also be harvested from radical prostatectomy specimens. Although the use of fresh tissues for practice is ideal, this can often be difficult to coordinate. Various techniques for the preservation of vasal segments have, therefore, been described. ${ }^{10}$ Specimens can be simply preserved in saline solution in the short term, but become 'macerated' over time. Alternatively, they may be frozen at $-20{ }^{\circ} \mathrm{C}$ after harvesting, but with variable post-thaw quality. Freezing the specimens in saline or glycerol is associated with better mucosal and muscularis quality than freezing without media. ${ }^{10}$ Belker et al. ${ }^{9}$ additionally noted that vasal segments preserved in saline-soaked gauze, in an airtight container, in the refrigerator, could be preserved for up to 8 weeks. Regardless of the preservation technique, it is important to remember to keep the vasal segments moistened with normal saline or lactated Ringer's irrigation when working under the microscope.

Although vasectomy segments can be used for either one-layer or two-layer vasovasostomy training, we recommend mastering the single-layer technique using silicone tubing and reserving vas specimens for practicing the more difficult multilayer anastomoses. Preparation of the vasal segments for re-anastomoses is similar to that described for the silicone tubing. A blue drape placed on the operating field acts as a contrast background. If the available vas segment is long in length, it should be secured in the Microspike approximator and sharply divided using a surgical knife between the arms of the approximator. If two shorter segments are used, the ends of these segments may need to be refreshed with the surgical knife prior to placement in the approximator. The lumen of the vas deferens should be carefully inspected under $\times 8$ to $\times 10$ magnification. If the lumen is not clearly visible, a fine microvessel dilator may be gently inserted into the lumen and removed. Care should be taken not to damage the mucosal layer. Following successful dilation, a mucosal ring can be seen. To enhance visualization of the mucosal ring, indigo carmine dye may also be applied to the cut surface of the vas using a Q-tip or Weck-Cel sponge (Beaver-Visitec Inc., Waltham, MA, USA). Indigo carmine is preferable to methylene blue as it is non-toxic to sperm. ${ }^{11}$ The microtip marking pen is then used to place six evenlyspaced marks around the circumference of the vas along both vasal ends. The two vasal ends are aligned with respect to these markings.

Double-armed 10-0 nylon suture is used to place three interrupted stitches along the anterior wall of the vas deferens, in through the mucosa, and out through the muscularis layer. Once these are securely tied, good approximation of the mucosal layer should be appreciated. Two to four interrupted 9-0 adventitial sutures are then placed in between the mucosal stitches to complete the second layer. The approximator is then turned over and the identical procedure is repeated on the posterior wall of the vas deferens (Figue 3). Six mucosal and 6-10 adventitial stitches are usually sufficient for achieving a watertight anastomosis. 
A successful vasal re-anastomosis is dependent on four basic principles: (i) the use of an operating microscope for optical magnification, and fine monofilament suture; (ii) preservation of the blood supply to the vas deferens; (iii) accurate mucosal approximation of the cut edges of the lumen of the vas deferens; and (iv) creation of a tension-free anastomosis. The long-term patency of a vaso-vasal anastomosis can, of course, only be evaluated in a live animal model. However, a technically accurate anastomosis using silicone tubing or vasal segments should be leak-proof when injected with fluid, and have a patent lumen when observed in cross-section under the microscope. Uniformity and accuracy of suture placement can also be assessed by longitudinally cutting open the mucosal and adventitial layers of the anastomosis to expose the lumen.

\section{Live animal surgery}

Six- to eight-week-old male Sprague-Dawley rats (200-300 g) are ideal for male infertility microsurgical training and research. Animal surgery requires access to an animal care facility, and must comply with the established guidelines for ethical conduct in the care and use of animals in research (www.iacuc.org). Compared to humans, the smaller size of the rat vas deferens and epididymis can be challenging to work with. However, this is the only model that allows for the practice of vasoepididymostomy techniques, and for the assessment of long-term anastomotic patency.

In our laboratory, we have developed an effective vasal occlusion model in rats. ${ }^{12}$ Compared to other techniques, occlusion of the vas deferens with two small metal clips, without division of the vas deferens, has the lowest incidence of sperm granuloma formation (approximately 50\%). Maximal epididymal tubule dilatation is observed seven days after vasal occlusion, and maintained thereafter. The rat vas deferens measures approximately $1.5-2 \mathrm{~mm}$ in outer diameter, and $0.15-0.25 \mathrm{~mm}$ in luminal diameter, compared to the $2 \mathrm{~mm}$ outer diameter and $0.5 \mathrm{~mm}$ luminal diameter for the human vas. Vasal occlusion, as described above, can achieve dilation of the testicular end of the vas deferens to a luminal diameter of $0.5 \mathrm{~mm}$, mimicking the discrepant luminal diameters seen in vasectomized patients seeking reversal. We have also used the unobstructed rat model for microsurgical training, which can be more demanding of the trainee in terms of technical perfection. In keeping with our institutional guidelines, anesthesia for all microsurgical procedures is induced using intraperitoneal injection of xylazine $\left(10 \mathrm{mg} \mathrm{kg}^{-1}\right)$ mixed with ketamine chloride $\left(100 \mathrm{mg} \mathrm{kg}^{-1}\right)$.

Rat vasovasostomy is performed as an end-to-end anastomosis using either a one-layer or two-layer technique, as described previously for silicone tubing and human vasal segments. Tissue feel in the rat model is certainly a more accurate reflector of the actual clinical experience. Hemostasis during infertility surgery in the animal model, as well as the operating room setting, should always be achieved with bipolar cautery, which creates a much smaller area of tissue damage than monopolar cautery.

Vasoepididymostomy represents the most challenging procedure in male infertility microsurgery. The success of this operation is heavily dependent on the quality and extent of surgical training and practice in the laboratory setting. Over the years, several different techniques have been described for vasoepididymostomy. ${ }^{13}$ Since 2002, we have adopted the longitudinal, end-to-side, two-suture intussusception technique as our standard method. ${ }^{14}$

Animals should be prepared for vasoepididymostomy by subjection to vasal obstruction, as described above. Following anesthesia induction, a midline incision allows easy access to the reproductive organs. The gubernaculum is divided for complete mobilization of the testis and epididymis. The cauda and corpus epididymis are gently separated from the testis, and the area of maximal epididymal tubular dilation is identified. A window is created in the tunic overlying this area, and the epididymal tubule of interested is identified. An anchoring stitch of 9-0 or 10-0 single armed suture is placed between the tunic and the vasal adventitia in order to decrease the pressure on the anastomosis. Using double-armed 10-0 nylon sutures, one needle from each of the two sutures is then placed longitudinally along both edges of the selected epididymal tubule, in a parallel fashion, without pulling through. A microknife or fine microscissors are used to open the epididymal tubule between the needles. Following this, the needles are pulled through the epididymal wall and prepared for placement through the vas. The needles are passed inside out from the vasal mucosa to the muscularis at the 2 o'clock, 4 o'clock, 8 o'clock and 10 o'clock positions. These positions should be pre-marked on the vas using the microdot marker. The sutures are then securely tied down, allowing the epididymal tubule to be intussuscepted within the lumen of the vas. Single armed 9-0 or 10-0 suture is then used to place additional interrupted stitches between the adventitia of the vas deferens and the tunic surrounding the epididymal tubule, in order to achieve a water-tight anastomosis (Figure 4).

The use of single-armed suture for vasoepididymostomy in the rat model has been previously described. ${ }^{6}$ This technique is best mastered in a controlled laboratory setting, but has wide applications in situations where double-armed suture is either unavailable or prohibitively expensive. Mechanical patency across the anastomosis following either technique can be demonstrated in the laboratory using a retrograde injection of indigo carmine into the vasal lumen, using a 27guage angiocatheter sheath (Figure 5).

\section{EVALUATION OF SURGICAL SKILLS}

Objective evaluation of skills developed in the microsurgical training laboratory is important for documenting the trainee's progress as well

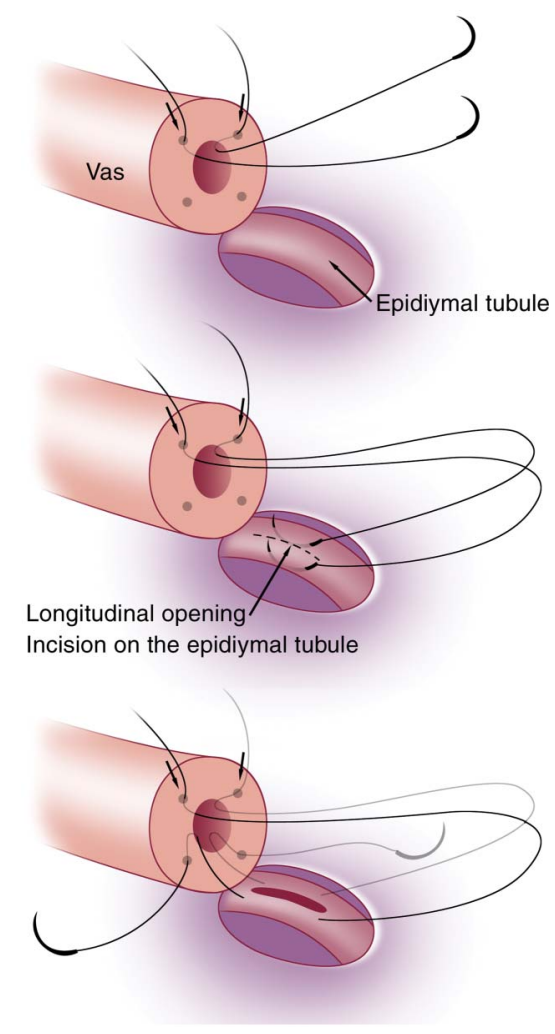

Figure 4 Longitudinal single-armed suture technique for vasoepididymostomy. 

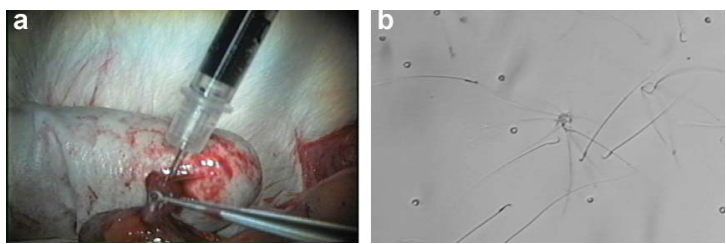

Figure 5 Mechanical patency (a) can be assessed at the end of the procedure by injection of saline or methylene blue across the anastomosis, while functional patency $(\mathbf{b})$ is assessed by a post-operative semen analysis.

as identifying areas that require further practice. Different methods of evaluating the technical skills gained by novice microsurgeons in the laboratory have been reported, ranging from direct observation and assessment by experts to the ability of surgeons to complete timed surgical drill tasks. ${ }^{1,3,15}$ In our laboratory, we use a detailed and systematic checklist to independently evaluate the various steps involved in performing a microsurgical anastomosis, such as handling of tissues and surgical suture, facility with tying secure and square knots, correctly setting up the anastomosis, and methodically executing the steps involved. A global rating scale to assess the surgeon's overall ability to plan and execute the operation is also evaluated. Together, these scales provide a comprehensive assessment of the individual's surgical ability.

\section{ROBOTIC-ASSISTED MICROSURGERY}

In some respects, microsurgery is ideally suited for adaptation to robotic-assisted surgery. Both fields rely on real-time, three-dimensional optical magnification, which results in some degree of hand and eye dissociation. Secondly, success in both types of surgical procedures relies heavily on surgical technique and accuracy of suture placement. ${ }^{16}$ Robotic assistance during microsurgery has the additional advantage of providing the surgeon with an ergonomic operating platform, and eliminating hand tremors. ${ }^{17}$ (Figure 6)

We first reported robotic rat vasovasostomy and vasoepididymostomy in $2004 .^{18}$ In this prospective trial, 24 rats were randomized to either the microsurgical or robotic surgery groups following vasectomy. At the 9-week follow-up period, no significant differences were noted in patency or sperm granuloma rates between the two groups. ${ }^{18}$ Since then, use of the DaVinci operating system for performing varicocelectomy, testicular sperm extraction, vasovasostomy and vasoepididymostomy has now been described in several series. Preliminary data, albeit limited, shows improved operative efficacy with comparable outcomes in terms of patency rates and semen parameters following vasectomy reversal. ${ }^{17}$

Currently, the cost of a robotic-assisted operation is significantly higher than that of the same procedure performed purely microsurgically. Larger clinical studies are still needed to better evaluate the truebenefit of robotic-assisted microsurgery. However, preliminary evidence supports an expanding role for robotics in urology and other microsurgery fields. As an example, this technology may allow experienced microsurgeons to perform microsurgical procedures in patients at remote locations where no experienced microsurgeons are available.

\section{SUMMARY}

Laboratory-based practice to enhance microsurgical skills improves the surgeon's confidence, and reduces stress and operating time, benefiting both the patient and the surgeon. Because success in male infertility surgery is so heavily dependent on surgical skills, and because patient outcomes are usually not apparent until weeks or months following surgery, it is imperative that cognitive and technical competency in microsurgery be achieved before entering the operating room.
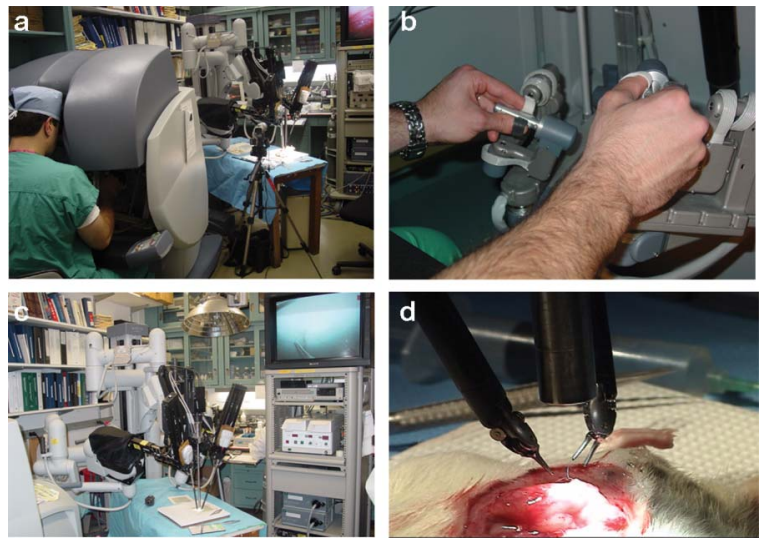

Figure 6 Set-up for robotic-assisted vasovasostomy. (a) surgeon at console, (b) hand stability, (c) docking of robot and (d) live animal surgery.

Establishing a microsurgical laboratory need not be expensive, and does not require a lot of elaborate equipment beyond an adequate operating microscope and select microsurgical instruments. Availability of such a facility enables advancement of surgical skills, and allows for research and experimentation, which, in turn, encourages innovation and advancement of the field of male infertility microsurgery.

\section{COMPETING FINANCIAL INTERESTS}

There are no competing financial interests for the authors of this manuscript.

1 Satterwhite T, Son J, Carey J, Zeidler K, Bari S et al. Microsurgery education in residency training: validating an online curriculum. Ann Plast Surg 2012; 68: 410-4.

2 Scallon SE, Fairholm DJ, Cochrane DD, Taylor DC. Evaluation of the operating room as a surgical teaching venue. Can J Surg 1992; 35: 173-6.

3 Grober ED, Hamstra SJ, Wanzel KR, Reznick RK, Matsumoto ED et al. Laboratory based training in urological microsurgery with bench model simulators: a randomized controlled trial evaluating the durability of technical skill. J Urol 2004; 172: 378-81.

4 Li PS, Schlegel PN, Goldstein M. Use of silicone medical grade tubing for microsurgical vasovasostomy training. Urology 1992; 39: 556-7.

5 Li PS, Ramasamy R, Goldstein M. Male Infertility Microsurgical Training. In: Sandlow JI, editor. Microsurgery for Fertility Specialists. New York: Springer; 2012.

6 Monoski MA, Schiff J, Li PS, Chan PT, Goldstein M. Innovative single-armed suture technique for microsurgical vasoepididymostomy. Urology 2007; 69: 800-4.

7 Goldstein M. Microspike approximator for vasovasostomy. J Urol 1985; 134: 74.

8 Goldstein M, Li PS, Matthews GJ. Microsurgical vasovasostomy: the microdot technique of precision suture placement. J Urol 1998; 159: 188-90.

9 Belker AM, Acland RD, Sexter MS, Roberts TL 3rd. Microsurgical two-layer vasovasostomy: laboratory use of vasectomized segments. Fertil Steril 1978; 29: 48-51.

10 Naughton CK, Thomas AJ Jr. Optimizing laboratory use of human vas deferens specimens for microsurgical practice. Urology 2002; 60: 320-3.

11 Sheynkin YR, Starr C, Li PS, Goldstein M. Effect of methylene blue, indigo carmine, and Renografin on human sperm motility. Urology 1999; 53: 214-7.

12 Young GP, Li PS, Goldstein M. Animal models for urologic microsurgical training and research. In: Goldstein M, editor. Surgery of Male Infertility. Philadelphia: W.B. Saunders Company; 1995.

13 Goldstein M. Surgical Management of Male Infertility. In: Wein A, Kavoussi LR, Novick AC, Partin AW, Peters CA, editors. Campbell-Walsh Urology. Philadelphia: Elsevier Saunders; 2012. p648-87.

14 Chan PT, Li PS, Goldstein M. Microsurgical vasoepididymostomy: a prospective randomized study of 3 intussusception techniques in rats. J Urol 2003; 169: 1924-9.

15 Brosious JP, Tsuda ST, Menezes JM, Baynosa RC, Stephenson LL et al. Objective evaluation of skill acquisition in novice microsurgeons. J Reconstr Microsurg 2012; 28: 539-42.

16 Schiff J, Li PS, Goldstein M. Robotic microsurgical vasovasostomy and vasoepididymostomy in rats. Int J Med Robot 2005; 1: 122-6.

17 Parekattil SJ, Brahmbhatt JV. Robotic approaches for male infertility and chronic orchialgia microsurgery. Curr Opin Urol 2011; 21: 493-9.

18 Schiff J, Li PS, Goldstein M. Robotic microsurgical vasovasostomy and vasoepididymostomy: a prospective randomized study in a rat model. J Urol 2004; 171: 1720-5. 